\title{
Neuroimaging characteristics of ruptured aneurysm as predictors of outcome after aneurysmal subarachnoid hemorrhage: pooled analyses of the SAHIT cohort
}

\author{
Blessing N. R. Jaja, MD, PhD, ${ }^{1-3}$ Hester Lingsma, PhD, ${ }^{5}$ Ewout W. Steyerberg, $\mathrm{PhD},{ }^{5}$ \\ Tom A. Schweizer, PhD, ${ }^{1-3}$ Kevin E. Thorpe, MMath, ${ }^{2,4}$ and R. Loch Macdonald, MD, PhD, ${ }^{1-3}$ \\ on behalf of the SAHIT investigators
}

${ }^{1}$ Division of Neurosurgery and ${ }^{2}$ Li Ka Shing Knowledge Institute, St. Michael's Hospital; ${ }^{3}$ nnstitute of Medical Science; ${ }^{4}$ Dalla Lana School of Public Health, University of Toronto, Toronto, Ontario, Canada; and ${ }^{5}$ Department of Public Health, Erasmus MC-University Medical Center Rotterdam, The Netherlands

\begin{abstract}
OBJECTIVE Neuroimaging characteristics of ruptured aneurysms are important to guide treatment selection, and they have been studied for their value as outcome predictors following aneurysmal subarachnoid hemorrhage (SAH). Despite multiple studies, the prognostic value of aneurysm diameter, location, and extravasated SAH clot on computed tomography scan remains debatable. The authors aimed to more precisely ascertain the relation of these factors to outcome.

METHODS The data sets of studies included in the Subarachnoid Hemorrhage International Trialists (SAHIT) repository were analyzed including data on ruptured aneurysm location and diameter $(7$ studies, $n=9125)$ and on subarachnoid clot graded on the Fisher scale (8 studies; $n=9452$ ) for the relation to outcome on the Glasgow Outcome Scale (GOS) at 3 months. Prognostic strength was quantified by fitting proportional odds logistic regression models. Univariable odds ratios (ORs) were pooled across studies using random effects models. Multivariable analyses were adjusted for fixed effect of study, age, neurological status on admission, other neuroimaging factors, and treatment modality. The neuroimaging predictors were assessed for their added incremental predictive value measured as partial $\mathrm{R}^{2}$.

RESULTS Spline plots indicated outcomes were worse at extremes of aneurysm size, i.e., less than 4 or greater than 9 $\mathrm{mm}$. In between, aneurysm size had no effect on outcome (OR 1.03, 95\% $\mathrm{Cl} 0.98-1.09$ for $9 \mathrm{~mm}$ vs $4 \mathrm{~mm}$, i.e., 75 th vs 25th percentile), except in those who were treated conservatively (OR 1.17, 95\% Cl 1.02-1.35). Compared with anterior cerebral artery aneurysms, posterior circulation aneurysms tended to result in slightly poorer outcome in patients who underwent endovascular coil embolization (OR 1.13, 95\% Cl 0.82-1.57) or surgical clipping (OR 1.32, 95\% Cl 1.10-1.57); the relation was statistically significant only in the latter. Fisher CT subarachnoid clot burden was related to outcome in a gradient manner. Each of the studied predictors accounted for less than $1 \%$ of the explained variance in outcome.

CONCLUSIONS This study, which is based on the largest cohort of patients so far analyzed, has more precisely determined the prognostic value of the studied neuroimaging factors. Treatment choice has strong influence on the prognostic effect of aneurysm size and location. These findings should guide the development of reliable prognostic models and inform the design and analysis of future prospective studies, including clinical trials.
\end{abstract}

http://thejns.org/doi/abs/10.3171/2015.4.JNS142753

KEY WORDS subarachnoid hemorrhage; intracranial aneurysm; outcome assessment; risk factors; meta-analysis; vascular disorders

$\mathrm{N}$ EUROIMAGING characteristics at hospital admission play an important role in the accurate diagnosis of subarachnoid hemorrhage (SAH) from ruptured intracranial aneurysms. ${ }^{3}$ They help to identify the aneurysm causing bleeding and outline its anatomical config- uration and that of adjoining structures to guide optimal choice of treatment modality for exclusion of the ruptured aneurysm from circulation. Neuroimaging parameters at hospital admission have been investigated for their value as predictors of outcome after SAH. Among these param-

ABBREVIATIONS ACA = anterior cerebral artery; CONSCIOUS-1 = Clazosentan to Overcome Neurological Ischemia and Infarction Occurring After SAH; D-SAT = University of Washington Database of Subarachnoid Treatment; GOS = Glasgow Outcome Scale; ICA = internal carotid artery; IQR = interquartile range; ISAT = International Subarachnoid Aneurysm Trial; MCA = middle cerebral artery; OR = odds ratio; RCT = randomized clinical trial; SAH = subarachnoid hemorrhage; SAHIT = Subarachnoid Hemorrhage International Trialists; SHOP = Subarachnoid Hemorrhage Outcomes Project; WFNS = World Federation of Neurosurgical Societies.

SUBMITTED December 4, 2014. ACCEPTED April 22, 2015.

INCLUDE WHEN CITING Published online October 23, 2015; DOI: 10.3171/2015.4.JNS142753. 
eters, subarachnoid clot burden on $\mathrm{CT}^{7}$ and aneurysm diameter and location have been the focus of many studies. ${ }^{15}$ Despite multiple studies, their value as outcome predictors is, to a large extent, debatable. The Fisher scale or its modified version or the Hijdra scale is often used to semiquantitatively assess CT clot burden. ${ }^{7,8,11}$ These scales are considered predictors of risk of delayed cerebral ischemia,${ }^{8}$ but no consensus exists as to their independent association with clinical outcomes after SAH. ${ }^{5,18-20,24,31,42,44}$ It is presently uncertain whether these neuroimaging parameters add incremental value beyond the effect of other established prognostic factors in SAH. With advances in diagnostic and interventional neuroradiology, and increasing patient selection for endovascular coiling rather than surgical clipping, further queries could be raised regarding their value as outcome predictors in SAH. ${ }^{17,46}$ Prognostic studies investigating neuroimaging characteristics have scarcely accounted for differences in treatment modality, ${ }_{19,31,34,38}$ though such studies may be helpful to better understand and more accurately evaluate the value of these characteristics as predictors of outcome after SAH.

We analyzed patient information in the largest repository of prospectively collected data on SAH patients, the Subarachnoid Hemorrhage International Trialists (SAHIT) repository, to investigate the prognostic value of SAH CT clot burden graded on the Fisher scale and ruptured aneurysm diameter and location. Specifically, we sought to more precisely ascertain their relationship to outcome of patients with SAH and assess how much added predictive information they provide beyond other established prognostic factors.

\section{Methods}

Details about the SAHIT repository have been published previously. ${ }^{14,21}$ In brief, the repository accrues data of randomized clinical trials (RCTs) and prospective cohort studies and hospital registries of SAH. For the present study, we pooled data on ruptured aneurysm diameter and location, which was available in 7 studies, and data on CT clot burden measured on the Fisher scale, which was available in 8 of the 14 studies in the repository. Fisher CT clot burden was estimated from the modified Fisher grade in the Columbia University Subarachnoid Hemorrhage Outcomes Project (SHOP) data set and from CT clot size (classified as thick or thin) and location (localized or diffused), with or without the presence of intraventricular hemorrhage in the tirilazad and Clazosentan to Overcome Neurological Ischemia and Infarction Occurring After SAH (CONSCIOUS-1) trials data sets. Ruptured aneurysm diameter was available as a continuous variable in 5 studies and as a categorical variable in the tirilazad and CONSCIOUS-1 data sets. We analyzed aneurysm diameter primarily as a continuous predictor, and secondarily as a categorical variable to include patients for whom data were dichotomized a priori, and examined for consistency in the results of both analyses. For the latter, we categorized aneurysm diameter as small (1-12 $\mathrm{mm})$, large (13$24 \mathrm{~mm}$ ), and giant ( $\geq 25 \mathrm{~mm}$ ). Ruptured aneurysm location was categorized broadly into anterior cerebral artery (ACA); internal carotid artery (ICA), including posterior communicating region; middle cerebral artery (MCA); and posterior circulation, inclusive of ruptured vertebral and basilar artery aneurysms. This categorization is identical to that used in the International Subarachnoid Aneurysm Trial (ISAT). ${ }^{25}$ The outcome measure was the score on the Glasgow Outcome Scale ${ }^{16}$ (GOS; with $1=$ death and $5=$ good recovery) at 3 months, which was imputed from the 2-month GOS score in the ISAT data and from the 6-month GOS score in the University of Washington Database of Subarachnoid Treatment (D-SAT). This technique is acceptable to minimize loss of data points, and it has been used previously in similar studies such as those of the International Mission on Prognosis and Clinical Trial Design in Traumatic Brain Injury (IMPACT) database. ${ }^{23,26}$

\section{Statistical Analysis}

The distribution of baseline characteristics was summarized by frequency tables for categorical variables and by the median and 25th and 75th percentiles or mean with standard deviation for continuous variables. Restricted cubic splines were used to study the relation between aneurysm diameter and GOS score. Splines are flexible polynomial functions that are very useful to demonstrate the shape of the relation of a continuous predictor to an outcome variable, where such relationship is anticipated to be nonlinear or complex..$^{41}$ Analysis of variance (1way ANOVA) was performed to test whether aneurysm diameter differed significantly with treatment modality. The unadjusted effect of the neuroimaging predictors was quantified using the technique of meta-analysis of individual participant data. First, proportional odds logistic regression models were fitted to obtain unadjusted odds ratios (ORs) with $95 \%$ confidence intervals by study. Next, the unadjusted ORs were pooled with a random effects model. Their consistency was illustrated across studies using forest plots and statistically tested using a test of heterogeneity. ${ }^{23}$ The adjusted effects of the neuroimaging parameters were assessed in a set of successive adjustment models using proportional odds logistic regression analysis, correcting for the fixed effect of study (Model A), age and World Federation of Neurosurgical Societies (WFNS) grade of neurological status at admission (Model B), other neuroimaging factors (Fisher CT clot burden, ruptured aneurysm location and diameter as applicable) (Model C), and treatment modality-whether ruptured aneurysm was repaired by clipping or coiling or treated conservatively (Model D, full adjustment model). We first illustrated relative prognostic strength with ORs. For aneurysm diameter, the OR was expressed as the change in interquartile range (IQR) (75th vs 25th percentile of aneurysm diameter), so we could directly compare the prognostic strength to those of categorical variables such as the Fisher grade. ${ }^{41}$ Second, we estimated the unique contribution of each variable in a model to outcome with partial $\mathrm{R}^{2}$ statistics. This statistic reflects the increase in explained variability and has been recommended as a means of assessing added incremental predictive value..$^{10,39}$

The proportion of missing data were small $(1.6 \%$ for analysis of the effect of aneurysm location and effect of aneurysm size, and 3\% for analysis of the effect of Fisher 
grade). To fill in missing data, we did multiple imputations by chained equations, generating 20 imputed data sets for analysis. The imputation models were specified on all covariates and GOS score and stratified by study. The following secondary analyses were performed. 1) Interactions: we tested a limited number of prespecified interaction effects including those of aneurysm diameter and location with patient age, neurological grade, and treatment modality to evaluate differential effects by these clinical characteristics; and 2) consistency over outcome splits: we fitted binary logistic models to assess the association between the predictor variables and outcome at each dichotomization split of the GOS (GOS 1 vs GOS $2-5$, GOS $1-2$ vs GOS $3-5$, etc.) to examine comparability of prognostic associations at each split point of the GOS. The proportional odds analysis provided a summary estimate over all splits rather than focusing on 1 specific split. The level of statistical significance was set at 5\%. The meta-analysis was performed in Stata version 12 (Statacorp). Other analyses were executed in the R platform ( $\mathrm{R}$ Foundation for Statistical Computation) using the rms and MICE libraries.

\section{Results}

\section{Patient and Aneurysm Characteristics}

The study cohort consisted of 9125 patients (derived from $5 \mathrm{RCT}$ data sets and 2 hospital registry data sets) for the analysis of the effect of ruptured aneurysm diameter and location, and 9452 patients (from 4 RCT data sets and 2 hospital registry data sets) for the analysis of the effect of Fisher CT clot burden. The median age of patients was 52 years (IQR 43-61 years). Median aneurysm diameter was $6 \mathrm{~mm}$ (IQR 4-9 mm). Mean aneurysm diameter significantly varied by treatment modality, with smaller aneurysms preferentially treated by coiling (coiling: $6.5 \pm$ $3.4 \mathrm{~mm}$; clipping: $8.01 \pm 5.6 \mathrm{~mm}$; none: $9.32 \pm 10.2 \mathrm{~mm}$; ANOVA $\mathrm{p}<0.001)$. Most aneurysms were in the anterior circulation (89\%). The distributions of Fisher CT clot burden, aneurysm diameter and location across studies are shown in Table 1. Small aneurysms were relatively more frequent in RCT studies than observational studies (73\%$96 \%$ vs $47 \%-67 \%$ ), whereas the converse was the case with respect to large diameter aneurysms $(3 \%-23 \%$ vs $9 \%-51 \%$ ). Posterior circulation aneurysms were relatively fewer in RCT data sets (3\%-14\%) than observational studies (18\%-19\%). Aneurysms of the ACA and MCA were preferentially treated by clipping while aneurysms of the posterior circulation were preferentially treated conservatively or with endovascular coiling (Table 2). A greater proportion of patients were classified as Fisher Grade 3 (42\%-80\% across studies; Table 1).

\section{Prognostic Associations}

The distribution of ruptured aneurysm diameter, location, and Fisher CT clot burden by 3-month GOS score is as shown in Table 3. Spline plots demonstrated a U-shaped relationship between aneurysm diameter and GOS score, with the inflection point at a diameter of $5.5 \mathrm{~mm}$ (Fig. 1). The unadjusted OR associated with the effect of aneurysm diameter and the effect of location was borderline across studies in the SAHIT repository, suggesting a weak relationship with outcome (Fig. 2). The pooled unadjusted OR for aneurysm diameter was 1.17 (95\% CI 0.97-1.41). Adjusting for the fixed effect of study, age, neurological status, aneurysm location, and Fisher grade had a slight effect on the OR (reduced from 1.13 to 1.09). On further accounting for treatment modality in the full adjustment model, the OR was 1.03 , which was statistically not significant (95\% CI 0.98-1.09). Table 4 shows that compared with ACA aneurysms, posterior circulation aneurysms were associated with $25 \%$ higher odds of poor outcome (OR 1.25, 95\% CI 1.08-1.44), adjusting for study effect, age, and WFNS grade. The effect was attenuated on further adjusting for treatment modality (OR 1.10, 95\% CI $0.95-1.28)$. We noted an interaction effect between aneurysm diameter and patient neurological status $(\mathrm{p}<0.001)$ and treatment modality $(p=0.005)$ but not between aneurysm diameter and age $(\mathrm{p}=0.226)$. We also noted a strong interaction between aneurysm location and treatment modality $(\mathrm{p}=0.0002)$ but none between aneurysm location

TABLE 1. Distribution of ruptured aneurysm location, diameter, and Fisher grade by study*

\begin{tabular}{|c|c|c|c|c|c|c|c|c|}
\hline Variable & $\begin{array}{c}C-1 \\
(n=433)\end{array}$ & $\begin{array}{c}\text { HHU } \\
(n=60)\end{array}$ & $\begin{array}{c}\text { IMASH } \\
(n=327)\end{array}$ & $\begin{array}{c}\text { IHAST } \\
(n=998)\end{array}$ & $\begin{array}{c}\text { ISAT } \\
(n=2143)\end{array}$ & $\begin{array}{c}\text { TIRILAZAD } \\
(\mathrm{n}=3552)\end{array}$ & $\begin{array}{c}\text { D-SAT } \\
(n=439)\end{array}$ & $\begin{array}{c}\text { SHOP } \\
(n=1500)\end{array}$ \\
\hline \multicolumn{9}{|l|}{ Location } \\
\hline ACA & $174(42)$ & $20(33)$ & - & $391(40)$ & $1085(51)$ & $1256(36)$ & $137(31)$ & $395(32)$ \\
\hline ICA & $118(29)$ & $11(18)$ & - & $309(31)$ & $697(32)$ & $1046(30)$ & $130(30)$ & $412(33)$ \\
\hline MCA & $76(18)$ & $21(35)$ & - & $205(21)$ & $303(14)$ & $711(20)$ & $88(20)$ & $209(17)$ \\
\hline$P C Q$ & $46(11)$ & $8(14)$ & - & $83(8)$ & $58(3)$ & $474(14)$ & $84(19)$ & $218(18)$ \\
\hline Diameter in mm, median (IQR) & - & $5(4-8)$ & - & $7(5-10)$ & $5(4-7)$ & - & $16(3-22)$ & $7(5-10)$ \\
\hline \multicolumn{9}{|l|}{ Fisher grade } \\
\hline 1 & 3 & 0 & $2(1)$ & $53(5)$ & $114(5)$ & $338(9)$ & $68(16)$ & $210(15)$ \\
\hline 2 & $68(16)$ & $2(3)$ & $24(7)$ & $342(34)$ & $360(17)$ & $455(13)$ & $47(11)$ & $315(22)$ \\
\hline 3 & $345(80)$ & $9(15)$ & $262(80)$ & $473(47)$ & 902 (42) & $2315(66)$ & $184(41)$ & $695(48)$ \\
\hline 4 & $17(4)$ & $49(81)$ & $39(12)$ & $130(13)$ & 753 (35) & $420(12)$ & $142(32)$ & 218 (15) \\
\hline
\end{tabular}

C-1 = CONSCIOUS 1 trial; HHU = Heinrich Heine University Concomitant Intraventricular Fibrinolysis and Low-Frequency Rotation after Severe Subarachnoid Hemorrhage Trial; IHAST = Intraoperative Hypothermia for Aneurysm Surgery Trial; IMASH = Intravenous Magnesium Sulphate for Aneurysmal Subarachnoid Hemorrhage trial; $P C Q=$ posterior circulation aneurysm; $-=$ data not available.

* Values are number (\%) unless otherwise indicated. 
TABLE 2. Distribution of aneurysm location by treatment modality*

\begin{tabular}{ccccc}
\hline & \multicolumn{3}{c}{ Treatment Modality } & \multirow{2}{*}{ Total } \\
\cline { 2 - 4 } Location & Clipping & Coiling & None & \\
\hline ACA & $2617(38.9)$ & $716(44.7)$ & $125(28.5)$ & $3458(39.5)$ \\
\hline ICA & $2096(31.2)$ & $501(31.3)$ & $126(28.7)$ & $2723(31.1)$ \\
\hline MCA & $1358(20.2)$ & $203(12.7)$ & $52(11.9)$ & $1613(18.4)$ \\
\hline PCQ & $653(9.7)$ & $182(11.4)$ & $136(30.9)$ & $971(11.1)$ \\
\hline
\end{tabular}

* Values are number $(\%)$.

and neurological status $(\mathrm{p}=0.52)$ or age $(\mathrm{p}=0.85)$. Motivated by the significant interaction effects, the full model (Model D) was stratified by treatment modality to obtain adjusted OR of aneurysm diameter and location for each treatment modality. We found that aneurysm diameter had no significant effect on outcome in patients who had undergone endovascular coiling (OR 0.92, 95\% CI 0.81$1.04)$, nor in those who had undergone surgical clipping (OR 1.04, 95\% CI 0.98-1.09). However, in patients who had neither (conservatively treated), the effect of aneurysm diameter was significant, with increasing diameter associated with poorer outcome (OR 1.17, 95\% CI 1.02-1.35). Compared with ACA aneurysms, those of the posterior circulation tended to result in poorer outcomes in patients who had undergone clipping (OR 1.32, 95\% CI 1.10-1.57) than in those who had undergone coiling (OR 1.13, 95\% CI 0.82-1.57), though the relationship was significant only in the former group. In patients who were treated conservatively, the outcome of posterior circulation aneurysms appeared better than that of ACA aneurysms (OR 0.37, 95\% CI 0.23-0.60).

In the unadjusted analysis, increasing Fisher grade of SAH on CT scan was associated with poorer outcome across studies (Fig. 3). The effect of Fisher grade varied significantly between studies, in particular the effects of Fisher Grades 3 and 4. In adjusted analysis (Table 4), increasing Fisher grade was independently related to poorer outcome in a gradient manner $(\mathrm{p}<0.001)$. In the full adjustment model (Model D), the OR associated with the ef-

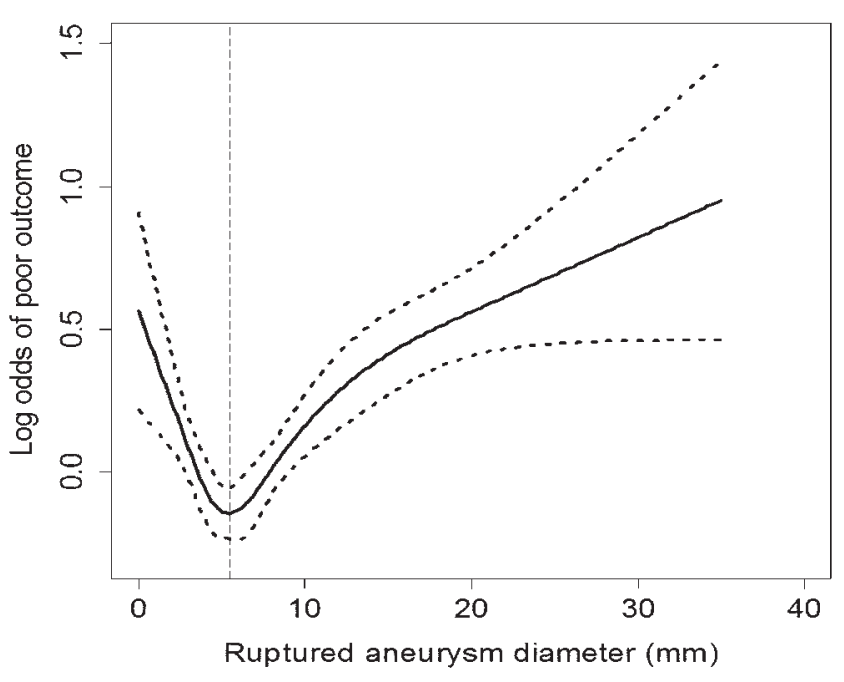

FIG. 1. Spline function showing a U-shaped relationship between aneurysm diameter and 3-month outcome on the GOS.

fect of Fisher Grade 2 was 1.26, Fisher Grade 3 was 1.77, and Fisher Grade 4 was 1.86, relative to Fisher Grade 1 as baseline category.

Figure 4 compares the relative prognostic strength of all predictors in the fully adjusted model (D), expressed as partial $\mathrm{R}^{2}$. We noted, that of all covariates, WFNS grade had the highest added predictive value, whereas the location of aneurysm had the least value. The value of treatment modality for predicting outcome was highest in patients with a poor grade. Aneurysm diameter, location, and Fisher grade had only small added value for predicting outcome (partial $\mathrm{R}^{2}<1 \%$ in each case). Secondary analysis showed prognostic effects were comparable at each dichotomization split point of the GOS.

\section{Discussion}

Increasing aneurysm size and lesion location in the posterior circulation were only weakly associated with outcome in this study. Their effects were strongly influenced

TABLE 3. Distribution of ruptured aneurysm location, diameter, and Fisher grade by GOS*

\begin{tabular}{|c|c|c|c|c|c|}
\hline Variable & Good & Moderate & Severe & Vegetative & Dead \\
\hline \multicolumn{6}{|l|}{ Location } \\
\hline ACA & $1629(49.8)$ & 727 (22.2) & $482(14.7)$ & $116(3.6)$ & 317 (9.7) \\
\hline ICA & $1330(52.5)$ & $544(21.5)$ & $316(12.4)$ & $58(2.3)$ & 286 (11.3) \\
\hline MCA & $749(49.9)$ & 327 (21.8) & $218(14.5)$ & $31(2.1)$ & 286 (11.3) \\
\hline$P C Q$ & $430(46.5)$ & $181(19.6)$ & $130(14.1)$ & $22(2.4)$ & $161(17.4)$ \\
\hline \multicolumn{6}{|l|}{ Diameter } \\
\hline $1-12 \mathrm{~mm}$ & 3519 (52.2) & 1473 (21.9) & $945(14.0)$ & $197(2.9)$ & 603 (8.9) \\
\hline $13-24 \mathrm{~mm}$ & $556(43.9)$ & $254(20.1)$ & 168 (13.3) & $27(2.1)$ & $262(20.7)$ \\
\hline$\geq 25 \mathrm{~mm}$ & $164(37.2)$ & $68(15.4)$ & $43(9.8)$ & $3(0.7)$ & $163(37.0)$ \\
\hline \multicolumn{6}{|l|}{ Fisher grade } \\
\hline 1 & $500(69.1)$ & $150(20.7)$ & $36(5.0)$ & $2(0.3)$ & $36(5.0)$ \\
\hline 2 & $950(62.9)$ & $330(21.8)$ & 141 (9.3) & $12(0.8)$ & $78(5.2)$ \\
\hline 3 & $2222(45.3)$ & $1048(21.4)$ & $724(14.8)$ & $155(3.2)$ & 757 (15.4) \\
\hline 4 & $679(41.7)$ & 341 (20.9) & $310(19.0)$ & $93(5.7)$ & 206 (12.7) \\
\hline
\end{tabular}

* Data on aneurysm diameter is presented when analyzed as continuous and as a categorical variable. Values are number (\%). 


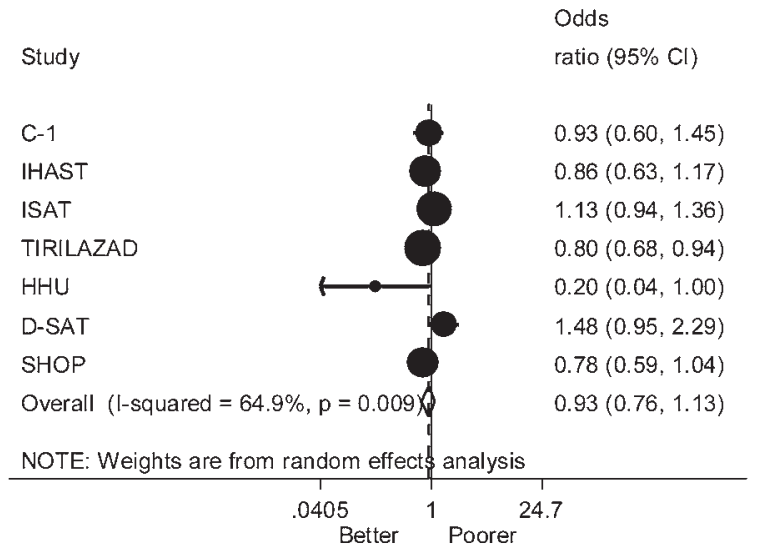

Internal carotid artery

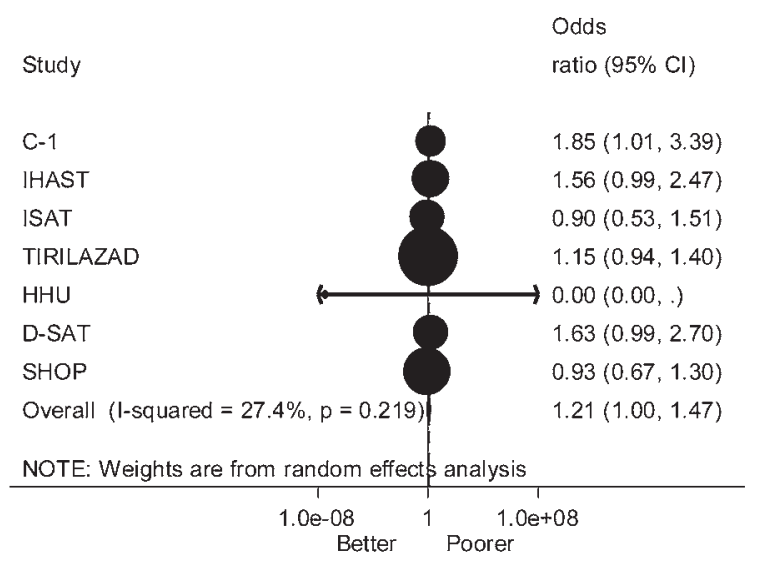

Posterior circulation

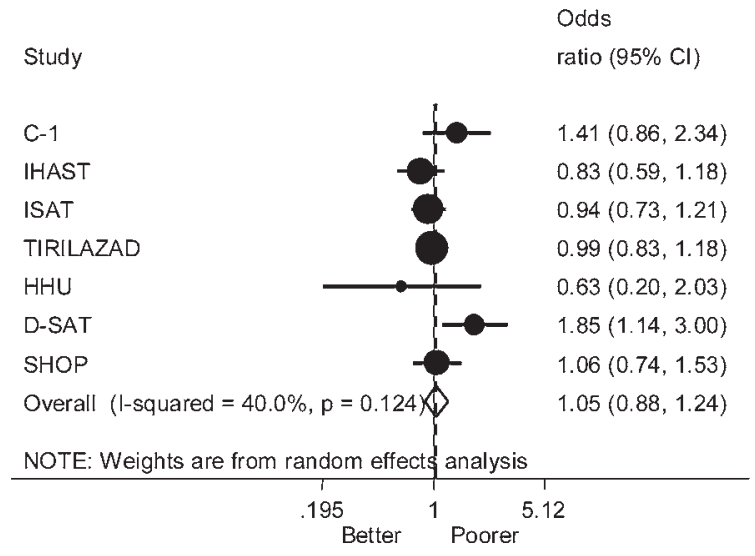

Middle cerebral artery

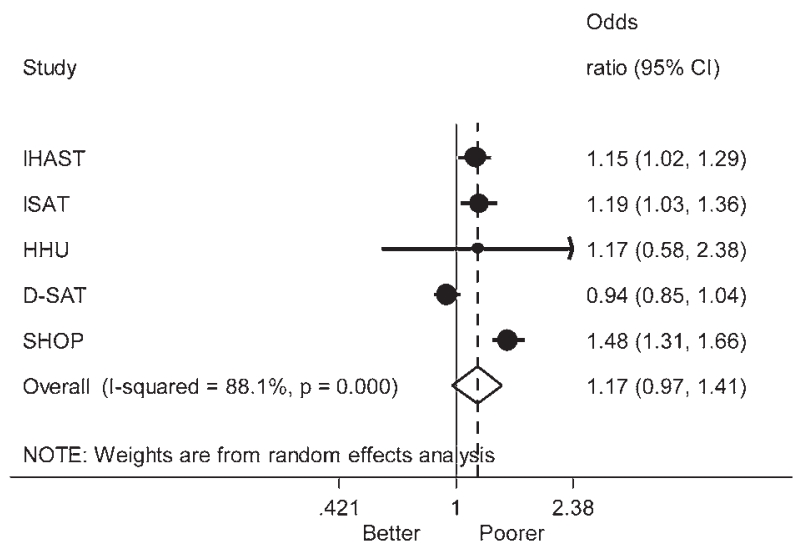

Aneurysm diameter ( $9 \mathrm{~mm}$ vs. $4 \mathrm{~mm}$ )

FIG. 2. Forest plots depicting unadjusted effect of ruptured aneurysm diameter and location across studies. C-1 = CONSCIOUS-1; $\mathrm{HHU}=$ Heinrich Heine University Concomitant Intraventricular Fibrinolysis and Low-Frequency Rotation after Severe Subarachnoid Hemorrhage Trial; IHAST = Intraoperative Hypothermia for Aneurysm Surgery Trial.

by treatment modality. Previous studies have identified aneurysm location as a predictor of periprocedural complication with MCA aneurysms at increased risk of intraoperative rupture. ${ }^{29}$ Some studies reported posterior circulation aneurysms to be associated with poorer outcomes than anterior circulation aneurysms, ${ }^{34,35}$ but these studies only included patients whose aneurysms were treated by surgical clipping. Studies in patients who had undergone coil embolization are scarcely available. Some researchers analyzed patients who had undergone coiling and clipping and found no significant relationship between aneurysm location and outcomes of patients with SAH. ${ }^{24,32,42}$ Some showed larger aneurysms correlated with higher risk of rebleeding ${ }^{9,22}$ and a higher risk of poor outcome, $9,12,18,24,31,33,34$ though at least 1 study $^{38}$ found no relationship between aneurysm size and outcome at 6 months on the GOS. A recent study involving 534 Japanese patients with data in a prospective registry also found no relationship between aneurysm size and outcome on the modified Rankin Scale after 12-months of follow-up. ${ }^{42}$ The inconsistent results of previous studies may be explained, in part, by the fact that they did not account for treatment modality. In the present analysis, the effects of aneurysm size and location varied with and was dependent on treatment modality. The prognostic effect of aneurysm size was only significant in patients who were treated conservatively, which suggests that appropriate selection of patients for treatment mitigates the effect of aneurysms size. The effect of posterior circulation aneurysms was less pronounced in patients who had undergone coiling than those who had undergone clipping, probably because these aneurysms were preferentially treated by coiling. In contrast, in their study of 914 patients, Ogilvy et al. ${ }^{28}$ noted a significant effect of posterior lesions relative to anterior lesions in patients who had undergone clipping but none in those who underwent coiling. That patients with posterior lesions who were treated conservatively in the present study seemed to experience better outcome relative to those with ACA lesions most likely reflects a treatment selection bias, as most posterior lesions with potentially poor outcomes would have had definitive intervention either by coiling or clipping, which further reinforces the need to account for treatment effect.

Comparing the prognostic strength of aneurysm size across previous studies is challenging as aneurysm size has been dichotomized differently with different threshold values applied in different studies, including the use of $10 \mathrm{~mm}, 9,18,29,3313 \mathrm{~mm},{ }^{24}$ or the use of different multiple categories. $^{34,38}$ We showed that aneurysm diameter has a 


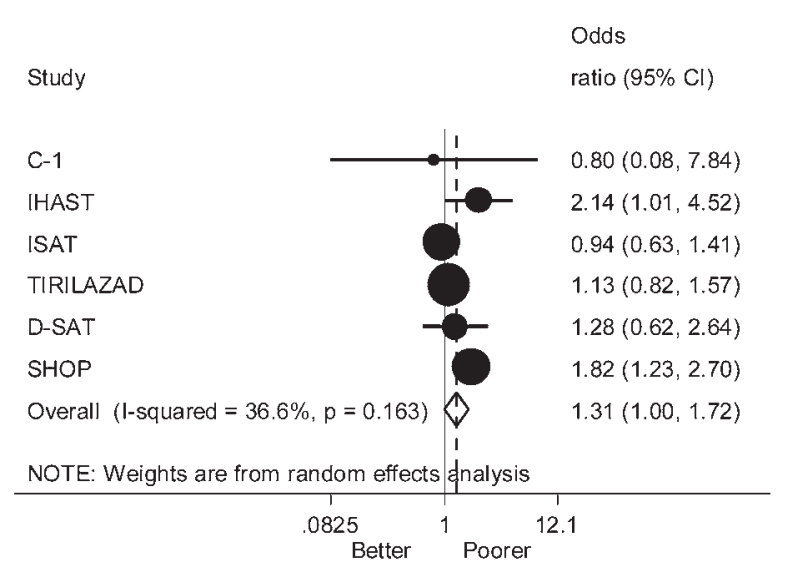

\section{Fisher Grade 2}

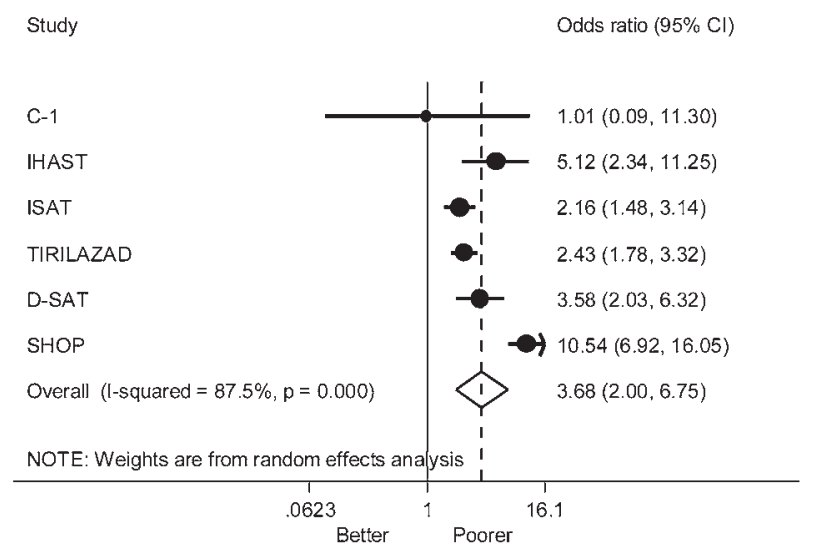

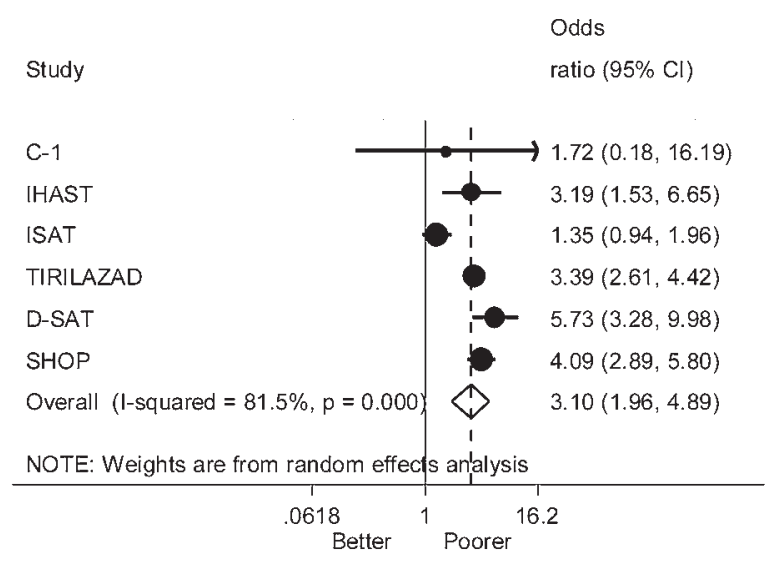

Fisher Grade 3

\section{Fisher Grade 4}

FIG. 3. Forest plots depicting unadjusted effect of Fisher grade across studies.

U-shaped relationship with outcome, a finding which suggests worse outcomes at extremes of size. Though this may be somewhat counterintuitive, it is not unlikely. Previous studies have reported that very small aneurysms are associated with more extensive hemorrhage,,$^{30,33,37,43}$ are more difficult to treat, are associated with a relatively higher risk of periprocedural complications, particularly when treated endovascularly, and have relatively higher morbidity and mortality. ${ }^{1,27,36,40}$ Giant aneurysms may present similar challenges. ${ }^{2,4,6}$ Furthermore, some researchers have suggested the possibility of an aggressive subset of small aneurysms that grow rapidly and rupture at very small size. ${ }^{14}$ If they exist, such aneurysms could represent a prognostically distinct group of intracranial aneurysms that theo-

TABLE 4. Relation of ruptured aneurysm location, size, and Fisher grade of CT clot burden to outcome*

\begin{tabular}{|c|c|c|c|c|}
\hline Variable & Model A & Model B & Model C & Model D \\
\hline \multicolumn{5}{|l|}{ Location } \\
\hline ACA & Referent & & & \\
\hline ICA & $0.91(0.76-1.09)$ & $1.01(0.91-1.12)$ & $1.00(0.90-1.11)$ & $0.99(0.89-1.10)$ \\
\hline MCA & $1.03(0.93-1.13)$ & $0.92(0.81-1.04)$ & $0.89(0.78-1.00)$ & $0.91(0.81-1.03)$ \\
\hline$P C Q$ & $1.17(1.04-1.32)$ & $1.25(1.08-1.44)$ & $1.20(1.04-1.39)$ & $1.10(0.95-1.28)$ \\
\hline Diameter (75th vs 25th percentile) & $1.13(1.08-1.19)$ & $1.10(1.05-1.15)$ & $1.09(1.04-1.15)$ & $1.03(0.98-1.09)$ \\
\hline \multicolumn{5}{|l|}{ Fisher grade } \\
\hline 1 & Referent & & & \\
\hline 2 & $1.48(1.28-1.77)$ & $1.17(0.96-1.41)$ & $1.21(1.00-1.47)$ & $1.26(1.04-1.53)$ \\
\hline 3 & $3.29(2.79-3.87)$ & $1.68(1.41-1.99)$ & $1.74(1.46-2.07)$ & $1.77(1.48-2.10)$ \\
\hline 4 & $3.89(3.26-4.64)$ & $1.75(1.45-2.11)$ & $1.79(1.48-2.17)$ & $1.86(1.54-2.26)$ \\
\hline
\end{tabular}

Model $\mathrm{A}=$ predictor $(\mathrm{CT}$ clot burden or aneurysm location or diameter $)+$ study; Model $\mathrm{B}=$ Model $\mathrm{A}+$ WFNS + age; Model $\mathrm{C}=$ Model $\mathrm{B}+$ neuroimaging data (Fisher grade + artery + ruptured aneurysm size, as applicable); Model $\mathrm{D}=$ Model $\mathrm{C}+$ repair (clipping vs coiling vs conservative).

* Analyses were done separately for each neuroimaging factor. Values are OR $(95 \% \mathrm{Cl})$. 


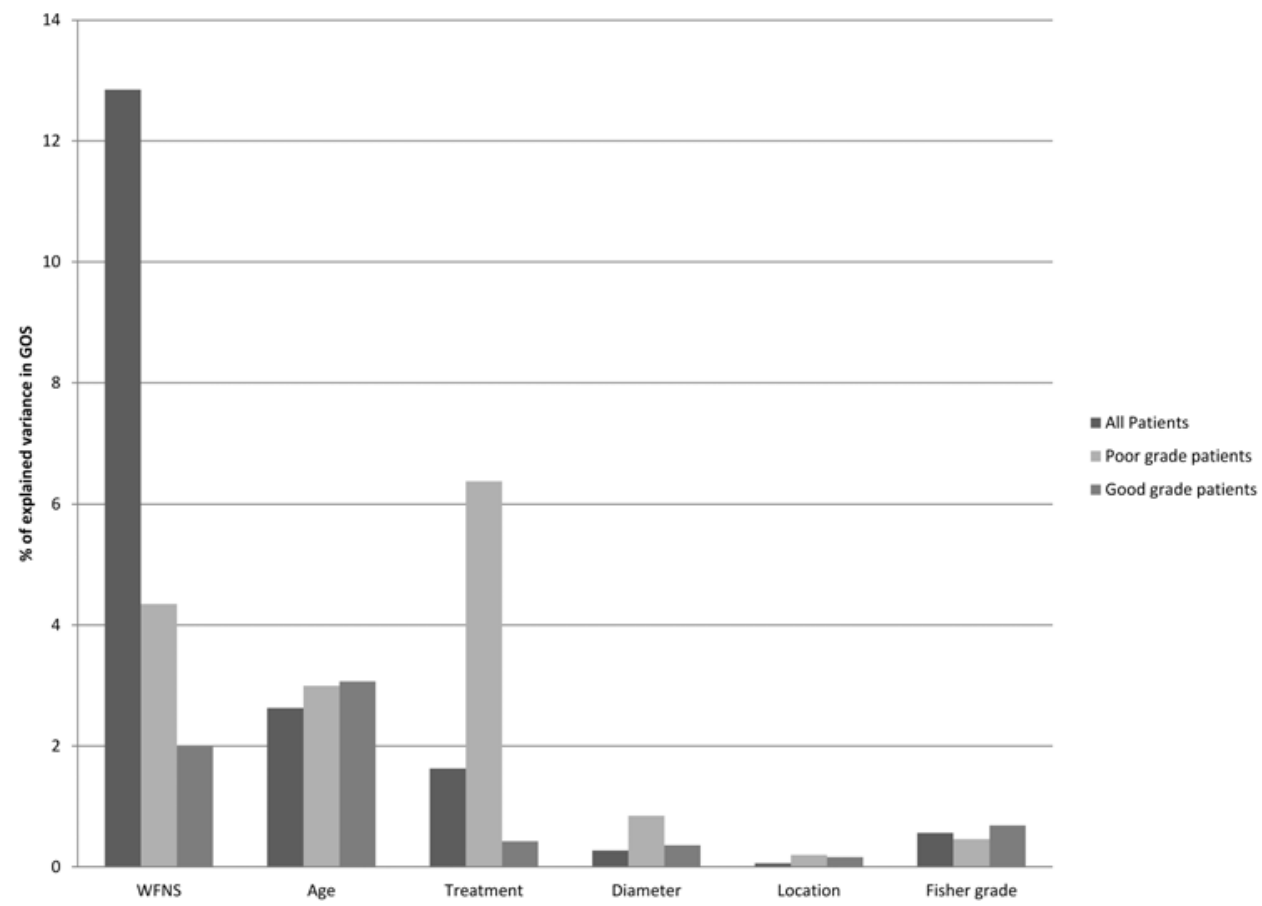

FIG. 4. Bar graph showing the relative prognostic value of studied neuroimaging predictor. Bars represent the difference in Nagelkerke's $\mathrm{R}^{2}$ values of adjustment models with and without the predictor.

retically could contribute to the poorer outcome of very small aneurysms seen in the present study.

Our finding that the Fischer grade is associated with outcome in a gradient manner agrees with a previous study ${ }^{31}$ though others found no gradient effect ${ }^{5}$ or no significant association between Fisher CT clot burden and outcome. ${ }^{19,24,42}$ The considerable between-study heterogeneity in the effect of Fisher CT clot burden, seen particularly for Fisher Grade 3 and 4, could be due to measurement variability associated with the subjective nature of the scale. ${ }^{17,45}$ This finding may also reflect our approximation of the Fisher grade in some studies from a simple categorization of clot thickness and location or from the modified Fisher scale. Nonetheless, in some respects, the results further underscore the need for better methods for grading subarachnoid blood volume, density, and distribution as seen on modern CT images.

In the present study, we have attempted a detailed analysis of the prognostic value of aneurysm size, location, and the Fisher grade in the SAHIT cohort, which far exceeds that of any previous studies. We envision that our data, in addition to helping us better understand the prognostic relevance of the studied variables, should be helpful to guide the development of reliable prognostic models and risk assessment tools for unruptured and ruptured aneurysms causing SAH. Such tools have potential to assist clinical practice and guide future trial design, particularly with respect to patient enrollment and data analysis. Though studies have inconsistently identified aneurysm size, location, and Fisher grade as prognostic factors after $\mathrm{SAH}$, most studies and our analysis of the SAHIT cohort agree that age and admission neurological status are the key prognostic factors among all factors studied to date.
The effect of age may be because of declining compensatory capacity of the aging brain to tolerate injury and the greater comorbid burden in older populations. Admission neurological status most likely reflects the severity of the primary injury and evolving secondary insults from multiple physiological mechanisms. Studies presenting prognostic models for SAH often include neuroimaging variables such as aneurysm size, location, and Fisher grade in addition to age and admission neurological status, but with little or no information as to how much incremental value was achieved to justify the added complexity of including the neuroimaging predictors. The limitations of this study include the fact that patient enrollment for some studies occurred prior to the adoption of endovascular coiling as first-line treatment modality in many centers, though the overall pattern of treatment is comparable to what is obtainable in many centers with expertise to treat SAH by surgical clipping or endovascular coiling. The generalizability of the findings may be affected by the preferential inclusion of RCT data sets, and potentially could have been improved had we included more data from all possible primary studies considering neuroimaging parameters and outcome after SAH. We also recognize that specific correlations and interactions between some variables may exist that we could not reliably evaluate, even with our large sample size. Despite the limitations, the study has a number of advantages: the large volume of prospectively collected data from multiple centers enhances the generalizability, and refined statistical analyses including proportional odds analysis recognize the ordinal nature of the GOS rather than loses information by dichotomizing the GOS as favorable and unfavorable outcome. 


\section{Conclusions}

In conclusion, this study provides empirical evidence that choice of treatment should be considered in evaluating the prognostic effect of aneurysm size and location. The effect is such that aneurysm size is associated with outcome only in patients who are conservatively treated. Aneurysm location has a weak effect on outcome in patients who are treated by clipping but no effect in patients who have undergone coiling. These findings should guide the development of reliable prognostic models and inform the design and analysis of future prospective studies, including clinical trials.

\section{Acknowledgments}

Our gratitude goes to Profs. M. Mamdani and G. Saposnik and Dr. Airton Leonardo de Oliveira Manoel for reading drafts of this manuscript and making useful comments to improve the clarity of the presentation. This work was supported by a grant from the Canadian Institutes for Health Research, Personnel Award from the Heart and Stroke Foundation of Canada, and an Early Researcher Award from the Ontario Ministry of Research and Innovation to Dr. T.A. Schweizer.

\section{References}

1. Brinjikji W, Murad MH, Lanzino G, Cloft HJ, Kallmes DF: Endovascular treatment of intracranial aneurysms with flow diverters: a meta-analysis. Stroke 44:442-447, 2013

2. Cantore G, Santoro A, Guidetti G, Delfinis CP, Colonnese C, Passacantilli E: Surgical treatment of giant intracranial aneurysms: current viewpoint. Neurosurgery 63 (4 Suppl 2):279-290, 2008

3. Connolly ES Jr, Rabinstein AA, Carhuapoma JR, Derdeyn CP, Dion J, Higashida RT, et al: Guidelines for the management of aneurysmal subarachnoid hemorrhage: a guideline for healthcare professionals from the American Heart Association/American Stroke Association. Stroke 43:1711-1737, 2012

4. Darsaut TE, Darsaut NM, Chang SD, Silverberg GD, Shuer LM, Tian L, et al: Predictors of clinical and angiographic outcome after surgical or endovascular therapy of very large and giant intracranial aneurysms. Neurosurgery 68:903915, 2011

5. de Toledo P, Rios PM, Ledezma A, Sanchis A, Alen JF, Lagares A: Predicting the outcome of patients with subarachnoid hemorrhage using machine learning techniques. IEEE Trans Inf Technol Biomed 13:794-801, 2009

6. Ding D, Starke RM, Evans AJ, Jensen ME, Liu KC: Balloon anchor technique for pipeline embolization device deployment across the neck of a giant intracranial aneurysm. J Cerebrovasc Endovasc Neurosurg 16:125-130, 2014

7. Fisher CM, Kistler JP, Davis JM: Relation of cerebral vasospasm to subarachnoid hemorrhage visualized by computerized tomographic scanning. Neurosurgery 6:1-9, 1980

8. Frontera JA, Claassen J, Schmidt JM, Wartenberg KE, Temes $\mathrm{R}$, Connolly ES Jr, et al: Prediction of symptomatic vasospasm after subarachnoid hemorrhage: the modified fisher scale. Neurosurgery 59:21-27, 2006

9. Guo LM, Zhou HY, Xu JW, Wang Y, Qiu YM, Jiang JY: Risk factors related to aneurysmal rebleeding. World Neurosurg 76:292-298, 253-254, 2011

10. Heinze G, Schemper M: Comparing the importance of prognostic factors in Cox and logistic regression using SAS. Comput Methods Programs Biomed 71:155-163, 2003

11. Hijdra A, Brouwers PJ, Vermeulen M, van Gijn J: Grading the amount of blood on computed tomograms after subarachnoid hemorrhage. Stroke 21:1156-1161, 1990
12. Inagawa T: Size of ruptured intracranial saccular aneurysms in patients in Izumo City, Japan. World Neurosurg 73:8492, e11, 2010

13. Jagadeesan BD, Delgado Almandoz JE, Kadkhodayan Y, Derdeyn CP, Cross DT III, Chicoine MR, et al: Size and anatomic location of ruptured intracranial aneurysms in patients with single and multiple aneurysms: a retrospective study from a single center. J Neurointerv Surg 6:169-174, 2014

14. Jaja BN, Attalla D, Macdonald RL, Schweizer TA, Cusimano MD, Etminan N, et al: The Subarachnoid Hemorrhage International Trialists (SAHIT) Repository: advancing clinical research in subarachnoid hemorrhage. Neurocrit Care 21:551-559, 2014

15. Jaja BN, Cusimano MD, Etminan N, Hanggi D, Hasan D, Ilodigwe D, et al: Clinical prediction models for aneurysmal subarachnoid hemorrhage: a systematic review. Neurocrit Care 18:143-153, 2013

16. Jennett B, Bond M: Assessment of outcome after severe brain damage. Lancet 1:480-484, 1975

17. Klimo P Jr, Schmidt RH: Computed tomography grading schemes used to predict cerebral vasospasm after aneurysmal subarachnoid hemorrhage: a historical review. Neurosurg Focus 21(3):E5, 2006

18. Lagares A, Gómez PA, Alen JF, Lobato RD, Rivas JJ, Alday $\mathrm{R}$, et al: A comparison of different grading scales for predicting outcome after subarachnoid haemorrhage. Acta Neurochir (Wien) 147:5-16, 2005

19. Lagares A, Gómez PA, Lobato RD, Alén JF, Alday R, Campollo J: Prognostic factors on hospital admission after spontaneous subarachnoid haemorrhage. Acta Neurochir (Wien) 143:665-672, 2001

20. Lindvall P, Runnerstam M, Birgander R, Koskinen LO: The Fisher grading correlated to outcome in patients with subarachnoid haemorrhage. Br J Neurosurg 23:188-192, 2009

21. Macdonald RL, Cusimano MD, Etminan N, Hanggi D, Hasan D, Ilodigwe D, et al: Subarachnoid Hemorrhage International Trialists data repository (SAHIT). World Neurosurg 79:418-422, 2013

22. Machiel Pleizier C, Algra A, Velthuis BK, Rinkel GJ: Relation between size of aneurysms and risk of rebleeding in patients with subarachnoid haemorrhage. Acta Neurochir (Wien) 148:1277-1280, 2006

23. McHugh GS, Butcher I, Steyerberg EW, Lu J, Mushkudiani $\mathrm{N}$, Marmarou A, et al: Statistical approaches to the univariate prognostic analysis of the IMPACT database on traumatic brain injury. J Neurotrauma 24:251-258, 2007

24. Mocco J, Ransom ER, Komotar RJ, Schmidt JM, Sciacca RR, Mayer SA, et al: Preoperative prediction of long-term outcome in poor-grade aneurysmal subarachnoid hemorrhage. Neurosurgery 59:529-538, 2006

25. Molyneux AJ, Kerr RS, Yu LM, Clarke M, Sneade M, Yarnold JA, et al: International subarachnoid aneurysm trial (ISAT) of neurosurgical clipping versus endovascular coiling in 2143 patients with ruptured intracranial aneurysms: a randomised comparison of effects on survival, dependency, seizures, rebleeding, subgroups, and aneurysm occlusion. Lancet 366:809-817, 2005

26. Mushkudiani NA, Engel DC, Steyerberg EW, Butcher I, Lu J, Marmarou A, et al: Prognostic value of demographic characteristics in traumatic brain injury: results from the IMPACT study. J Neurotrauma 24:259-269, 2007

27. Nguyen TN, Raymond J, Guilbert F, Roy D, Bérubé MD, Mahmoud M, et al: Association of endovascular therapy of very small ruptured aneurysms with higher rates of procedure-related rupture. J Neurosurg 108:1088-1092, 2008

28. Ogilvy CS, Cheung AC, Mitha AP, Hoh BL, Carter BS: Outcomes for surgical and endovascular management of intracranial aneurysms using a comprehensive grading system. Neurosurgery 59:1037-1043, 2006 
29. Pierot L, Cognard C, Anxionnat R, Ricolfi F: Ruptured intracranial aneurysms: factors affecting the rate and outcome of endovascular treatment complications in a series of 782 patients (CLARITY study). Radiology 256:916-923, 2010

30. Qureshi AI, Sung GY, Suri MF, Straw RN, Guterman LR, Hopkins LN: Factors associated with aneurysm size in patients with subarachnoid hemorrhage: effect of smoking and aneurysm location. Neurosurgery 46:44-50, 2000

31. Risselada R, Lingsma HF, Bauer-Mehren A, Friedrich CM, Molyneux AJ, Kerr RS, et al: Prediction of 60 day case-fatality after aneurysmal subarachnoid haemorrhage: results from the International Subarachnoid Aneurysm Trial (ISAT). Eur J Epidemiol 25:261-266, 2010

32. Risselada R, Lingsma HF, Molyneux AJ, Kerr RS, Yarnold J, Sneade M, et al: Prediction of two month modified Rankin Scale with an ordinal prediction model in patients with aneurysmal subarachnoid haemorrhage. BMC Med Res Methodol 10:86, 2010

33. Roos EJ, Rinkel GJE, Velthuis BK, Algra A: The relation between aneurysm size and outcome in patients with subarachnoid hemorrhage. Neurology 54:2334-2336, 2000

34. Rosen DS, Macdonald RL: Grading of subarachnoid hemorrhage: modification of the World Federation of Neurosurgical Societies scale on the basis of data for a large series of patients. Neurosurgery 54:566-576, 2004

35. Rosengart AJ, Schultheiss KE, Tolentino J, Macdonald RL: Prognostic factors for outcome in patients with aneurysmal subarachnoid hemorrhage. Stroke 38:2315-2321, 2007

36. Rosen $\varnothing \mathrm{rn} \mathrm{J}$, Eskesen V: Patients with ruptured intracranial saccular aneurysms: clinical features and outcome according to the size. Br J Neurosurg 8:73-78, 1994

37. Russell SM, Lin K, Hahn SA, Jafar JJ: Smaller cerebral aneurysms producing more extensive subarachnoid hemorrhage following rupture: a radiological investigation and discussion of theoretical determinants. J Neurosurg 99:248-253, 2003

38. Salary M, Quigley MR, Wilberger JE Jr: Relation among aneurysm size, amount of subarachnoid blood, and clinical outcome. J Neurosurg 107:13-17, 2007

39. Schemper M: Predictive accuracy and explained variation. Stat Med 22:2299-2308, 2003

40. Signorelli F, Scholtes F, Bojanowski MW: [Very small intracranial aneurysms: Clip or coil.] Neurochirurgie 58:156159, $2012(\mathrm{Fr})$

41. Steyerberg E: Clinical Prediction Models: A Practical Approach to Development, Validation and Updating. New York: Springer, 2009

42. Taki W, Sakai N, Suzuki H: Determinants of poor outcome after aneurysmal subarachnoid hemorrhage when both clipping and coiling are available: Prospective Registry of Subarachnoid Aneurysms Treatment (PRESAT) in Japan. World Neurosurg 76:437-445, 2011

43. Taylor CL, Steele D, Kopitnik TA Jr, Samson DS, Purdy PD: Outcome after subarachnoid hemorrhage from a very small aneurysm: a case-control series. J Neurosurg 100:623-625, 2004

44. Turck N, Vutskits L, Sanchez-Pena P, Robin X, Hainard A, Gex-Fabry M, et al: A multiparameter panel method for outcome prediction following aneurysmal subarachnoid hemorrhage. Intensive Care Med 36:107-115, 2010

45. van Norden AG, van Dijk GW, van Huizen MD, Algra A, Rinkel GJ: Interobserver agreement and predictive value for outcome of two rating scales for the amount of extravasated blood after aneurysmal subarachnoid haemorrhage. J Neurol 253:1217-1220, 2006

46. Wilson DA, Nakaji P, Abla AA, Uschold TD, Fusco DJ, Oppenlander ME, et al: A simple and quantitative method to predict symptomatic vasospasm after subarachnoid hemorrhage based on computed tomography: beyond the Fisher scale. Neurosurgery 71:869-875, 2012

\section{Disclosure}

Dr. Macdonald reports ownership of Edge Therapeutics, Inc. and receiving non-study-related clinical or research effort support from the Brain Aneurysm Foundation, Heart and Stroke Foundation of Canada, Canadian Institutes for Health Research, and Physicians Services Incorporated Foundation.

\section{Author Contributions}

Conception and design: all authors. Acquisition of data: Macdonald, Jaja. Analysis and interpretation of data: Jaja, Lingsma, Steyerberg, Thorpe. Drafting the article: Jaja. Critically revising the article: all authors. Reviewed submitted version of manuscript: all authors. Approved the final version of the manuscript on behalf of all authors: Macdonald. Statistical analysis: Jaja. Administrative/technical/material support: Jaja, Schweizer. Study supervision: Macdonald, Steyerberg, Thorpe.

\section{Correspondence}

R. Loch Macdonald, Department of Surgery, University of Toronto, 30 Bond St., Toronto, ON M5B 1W8, Canada. email: macdonaldlo@smh.ca. 